\title{
High-risk KPC-producing Klebsiella pneumoniae lack type I R-M systems
}

\author{
Ying Zhou ${ }^{\mathrm{a}}$, Dongxing Tian ${ }^{\mathrm{a}}$, Yu Tang ${ }^{\mathrm{b}}$, Lianhua $\mathrm{Yu}^{\mathrm{c}}$, Yunkun Huang ${ }^{\mathrm{d}}$, Gang $\mathrm{Li}^{\mathrm{e}}$, Meng $\mathrm{Li}^{\mathrm{f}}$, \\ Yong Wang ${ }^{g}$, Zehua Yang ${ }^{\mathrm{h}}$, Laurent Poirel ${ }^{\mathrm{i}, \mathrm{j}, * *}$, Xiaofei Jiang ${ }^{\mathrm{a}, *}$ \\ a Department of Laboratory Medicine, Huashan Hospital, Shanghai Medical College, Fudan University, Shanghai, China \\ ${ }^{\mathrm{b}}$ Department of Laboratory Medicine, Shanghai Chest Hospital, Shanghai Jiao Tong University, Shanghai, China \\ ${ }^{\mathrm{c}}$ Department of Laboratory Medicine, Taizhou Municipal Hospital, Taizhou, China \\ d Department of Laboratory Medicine Kunming Yan'an Hospital, Kunming, China \\ ${ }^{\text {e }}$ Department of Laboratory Medicine, Jinshan Hospital, Shanghai Medical College, Fudan University, Shanghai, China \\ ${ }^{\mathrm{f}}$ Department of Clinical Laboratory, The First Affiliated Hospital of Guangxi Medical University, Nanning, Guangxi, China \\ g Department of Clinical Laboratory, Shandong Provincial Hospital affiliated to Shandong University, Jinan, Shandong, China \\ ${ }^{\mathrm{h}}$ Department of Laboratory Medicine, Sixth Hospital of Shanxi Medical University, Taiyuan, China \\ ${ }^{i}$ Emerging Antibiotic Resistance Unit, Medical and Molecular Microbiology, Department of Medicine, University of Fribourg, Fribourg, Switzerland \\ ${ }^{\mathrm{j}}$ Laboratoire Europeen ASSOCIE (LEA) INSERH, IAME (Paris, France), University of Fribourg, Fribourg, Switzerland
}

Key words:

Type I R-M systems

bla

Carbapenem-resistant Klebsiella pneumoniae

Klebsiella pneumoniae clonal group 258

\begin{abstract}
A B S T R A C T
Klebsiella pneumoniae carbapenemase (KPC)-producing K. pneumoniae (KPC-KP) have disseminated worldwide and are a major threat to public health. The multidrug-resistant (MDR)-phenotype of KPC-KP are commonly associated with the presence of high molecular weight $b a_{\mathrm{KPC}}$ plasmids. Restrictionmodification (R-M) systems provide bacteria with innate defense against plasmids or other infectious gene elements. As bla $a_{\mathrm{KPC}}$ plasmids are favored by such MDR K. pneumoniae, it was of interest to examine the co-distribution of R-M and acquired bla $a_{\mathrm{KPC}}$ plasmids in KPC-KP. A total of 459 clinical K. pneumoniae isolates in China and 217 global whole-genome sequences in GenBank were collected to determine the prevalence of type I R-M systems. The type I R-M systems were scarce in the KPC-positive group and high-risk Klebsiella pneumoniae clonal group 258 (CG258). The polymorphisms of type I R-M observed in K. pneumoniae revealed the ubiquity of their recognition sequences in DNA; therefore, the type I R-M systems could attack most invading DNA elements, such as $b a_{\mathrm{KPC}}$ genes. Overall, this work indicated the type I R-M systems may impact the acquisition of bla $_{\mathrm{KPC}}$ genes in K. pneumoniae.
\end{abstract}

\section{Introduction}

Klebsiella pneumoniae carbapenemase (KPC)-producing K. pneumoniae (KPC-KP) were first identified in 2001 [1] and have become important nosocomial pathogens and a global public health concern because of their prevalence and the associated high rate of mortality $[2,3]$. Given the success of the high-risk KPC-KP worldwide, identifying factors contributing to the epidemic success of these isolates becomes an important public health question.

Multidrug-resistant (MDR) K. pneumoniae appear to easily acquire antibiotic resistance genes associated with mobile genetic elements (MGEs) by horizontal gene transfer (HGT) [4,5]. Previous

\footnotetext{
* Corresponding author: Xiaofei Jiang, Tel: +86-021-52888316.

** Co-correspondent

E-mail addresses: laurent.poirel@unifr.ch (L. Poirel), Jiangxi2154@sina.com (X. Jiang).
}

studies have demonstrated that prokaryotic immune systems are able to actively resist infectious MGEs with a high degree of selectivity [6], thus we proposed that such multidrug species (KPC-KP) may lack or have lost endogenous barriers to HGT, so they more easily acquire MGEs.

Restriction-modification (R-M) systems provide a form of genome defense by acting as barriers to HGT through selfrecognition vs. non-self-recognition of methylation signatures [6,7]. $\mathrm{R}-\mathrm{M}$ systems are classified into four different types based on the specific number and types of enzymes in the system [8]. This study focused on the type I R-M systems [7,9]. Another three systems (II/II//IV) identified in K. pneumoniae were not as structurally complete and widely distributed as type I R-M (Supplementary data 3a).

A type I restriction enzyme comprises three subunits encoded by three closely linked genes, $h s d R$, hsdM, and hsdS. The HsdR subunit (encoding by $h s d R$ ) is required for restriction [7,9-11]. The 
HsdM subunits encoded by hsdM are sufficient for methyltransferase activity, which modifies these sites through methylation to prevent cleavage. Notably, the HsdS subunit is essential for conferring target sequence specificity to both the restriction and modification activities of the complex [7,9]. Interestingly, in addition to the complete complex comprising all three subunits (HsdRMS), a complex with two subunits that lacks HsdR (HsdMS) is also functional and has only methyltransferase activity $[7,9]$.

Although the type I R-M system may limit entry of mobile elements, the epidemiological data supporting the role of such a system in swiftly spreading high-risk KPC-KP are still insufficient. In this study, the correlation between these systems and $b l a_{\mathrm{KPC}}-$ positive isolates was explored. The distribution of both type I R$\mathrm{M}$ systems and bla $a_{\mathrm{KPC}}$ genes in K. pneumoniae was studied, with the aim to clarify the relationship between the activity of the system and $b l a_{\mathrm{KPC}}$ dissemination in K. pneumoniae. All type I R-M systems obtained from the NCBI databases were categorized and analysed to determine their diversity and universality in K. pneumoniae. Overall, this study was a first step towards understanding the role of R-M in regulating HGT in K. pneumoniae and the potential for R$\mathrm{M}$ as one mechanism for the globally successful dissemination of KPC-KP isolates.

\section{Materials and Methods}

\subsection{Clinical isolates}

A total of 459 non-duplicated K. pneumoniae isolates were randomly collected from individual patients at seven hospitals in six provinces of China (Fig. S1, Supplementary data 1) from January 2017 to February 2018. Among these, 247 carbapenem-resistant K. pneumoniae (CR-KP) and 212 carbapenem-sensitive K. pneumoniae (CS-KP) were collected contemporaneously from similar departments (Table S2, Supplementary data 1). Identification and antimicrobial susceptibility testing were performed on the VITEK 2 system (bioMerieux). The presence of $b_{1} a_{\mathrm{KPC}}$ gene about these strains was determined by the pair of primers listed in Table S1. Multilocus sequence typing (MLST) was performed according to the protocol described on the Pasteur Institute MLST website for K. pneumoniae (http://bigsdb.pasteur.fr/klebsiella/klebsiella.html).

\subsection{Genome and type I R-M complex analysis}

All the K. pneumoniae complete genome sequences publicly available (217 in total) and all the type I R-M systems harbored in these strains were downloaded from GenBank in April 2018 (Supplementary data 3a). Plasmid incompatibility type was identified by comparing with information in the plasmid MLST locus/sequence definitions database (https://pubmlst.org/bigsdb?db= pubmlst_plasmid_seqdef). All the information about the components of type I R-M systems were obtained from the REBASE database (http://rebase.neb.com) [12]. According to the difference of R-M units and whether it was carried by plasmid [8], the type I R-M systems detected in 217 complete genome sequences of $K$. pneumoniae were divided into four families (IA/B/C/D) (Fig. 2 and Fig. S2, Supplementary data 1). The software MEGA 5 [13] was used to perform a Maximum Likelihood-based phylogenetic tree (Jukes-Cantor model) based on aligned amino acid sequences of HdsM of RM-positive K. pneumoniae genomes (Supplementary data 2).

\subsection{PCR screening of type I R-M systems}

Due to the absence of HsdR protein in some type I R-M systems (HsdMS), the HsdM protein was targeted for the homology analysis of type I R-M systems identified in K. pneumoniae. All the type I R$\mathrm{M}$ identified in the 217 complete genome sequences of K. pneumoniae available in GenBank can be classified in 14 major clades, and clade 4, 6 and 7 are plasmid-borne, after the analysis of HdsM protein through the software MEGA 5 (Supplementary data 2). Thus, 14 pairs of primers obtained from GenBank were designed to test the presence of type I R-M systems and distinguish whether they are linked to plasmids (clade 4, 6 and 7). The collected clinical isolates were tested for the presence of R-M complex by analytical PCR using the primers listed in Table S1.

\subsection{Statistics}

Statistical significance was assessed using a two-tailed Student's t-test, one-way analysis of variance (ANOVA) test, or chi-square test of the GraphPad Prism8 software.

\section{Results}

\subsection{Type I R-M systems are extremely rare in KPC-KP}

A total of $459 \mathrm{~K}$. pneumoniae isolates were collected from six provinces in China. In parallel, $217 \mathrm{~K}$. pneumoniae whole-genome sequences from worldwide and GenBank databases were analysed to determine the distribution of the type I R-M systems in K. pneumoniae. The type I R-M unit was extremely rare in the KPC-positive isolates, whereas the unit was abundant among the KPC-negative isolates, either within the Chinese collection of clinical isolates (43/247 vs. $122 / 212, P<0.0001)$ or within the Genbank database (33/75 vs. $80 / 142, P<0.0001$ ) (Fig. $1 \mathrm{~A}$ ). Intriguingly, most KPCpositive strains obtained the type I R-M systems from the plasmid, whereas in the KPC-negative group, the majority harbored these immune systems naturally. Notably, these plasmid-borne type I R$\mathrm{M}$ units always coexist with the antibiotic elements such as bla $a_{\mathrm{KPC}}$ gene on the same plasmid (Supplementary data $3 \mathrm{~b}$ ), which would protect these antibiotic DNA from attacking the same REase (Supplementary data $3 \mathrm{~b}$ ). Together, these results indicated an epidemic link between type I R-M systems and $K$. pneumoniae with $b l a_{\mathrm{KPC}}$.

\subsection{High-risk CG258 K. pneumoniae lacks type I R-M systems but is rich in bla $\mathrm{KPC}_{\mathrm{K}}$ genes}

In this study, the epidemic of bla $a_{\mathrm{KPC}}$ was primarily restricted to CG258 K. pneumoniae as shown in previous studies [14] (Fig. 1B). Thus, the CG258 lineage may be immunocompromised strains lacking type I R-M systems. The co-distribution of R-M and acquired bla $_{\mathrm{KPC}}$ genes was investigated in different $K$. pneumoniae clone groups, including high-risk CG258 group, MDR group (CG15, CG147 and CG29), hypervirulent group (CG23 and CG65) and others (Fig. 1C). Type I R-M systems were extremely rare in CG258 lineages, either within the Chinese collection of clinical isolates or within the Genbank database. Moreover, a highly significant inverse correlation was found between the presence of type I R-M units and bla ${ }_{K P C}$ genes in all K. pneumoniae clone groups. These findings indicated the lack of robust type I R-M systems in the CG258 group may be related to the $b l a_{\mathrm{KPC}}$ genes dissemination in such a high-risk lineage.

\subsection{Type I R-M systems are pleomorphic in $\mathrm{K}$. pneumoniae}

Type I R-M systems were widespread among the K. pneumoniae, particularly in the KPC-negative group (Table S2 and Supplementary data 3a). The structure and function of these diverse systems were further analysed. A variety of mobile genetic units were found to be linked with R-M gene complexes in $K$. pneumoniae 
A
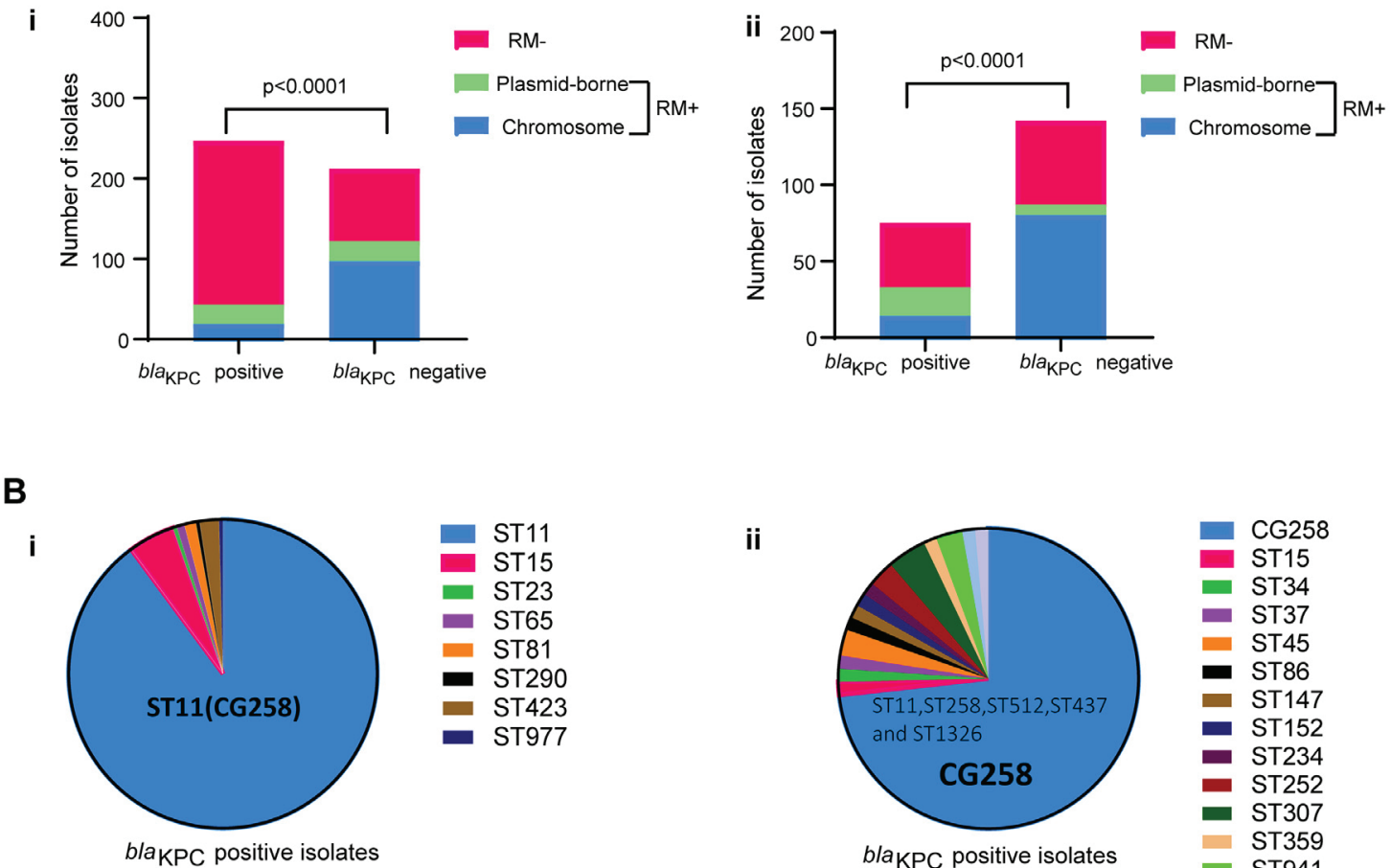

ii

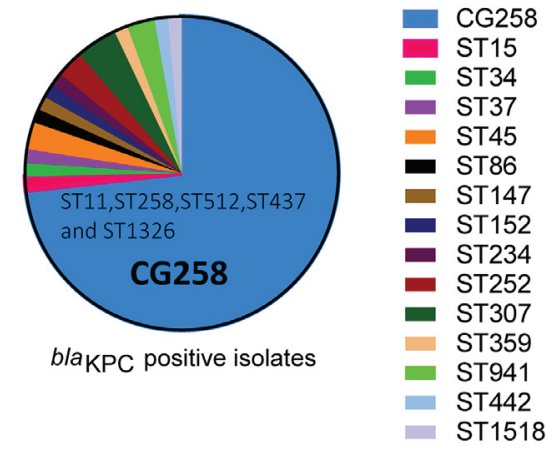

C
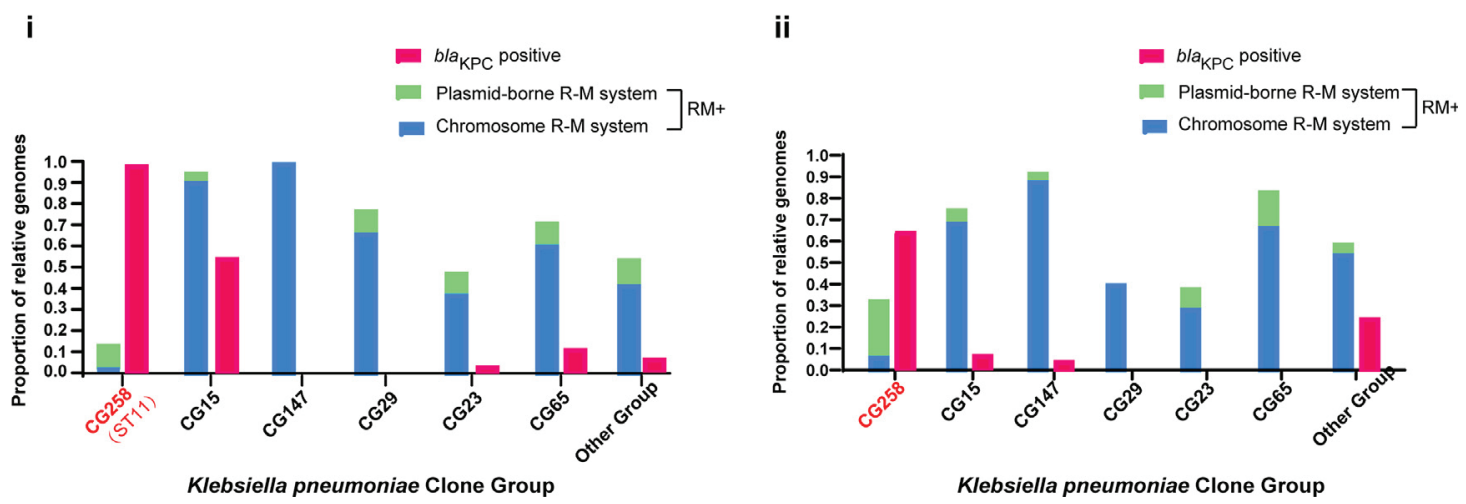

Fig. 1. Presence of type I R-M systems in the bla $a_{\mathrm{KPC}}$-positive/-negative groups and CG258 group/other clone groups of K. pneumoniae. (A) i. Presence of type I R-M systems in 459 Chinese clinical isolates collected in this study; ii. Presence of type I R-M systems in 217 completely sequenced strains available in GenBank. (B) i. MLSTs of bla $a_{\mathrm{KP}-}-$

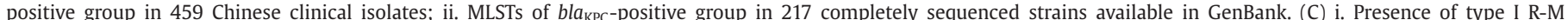
systems in 459 Chinese clinical isolates collected in this study; ii. Presence of type I R-M systems in 217 completely sequenced strains available in GenBank. $P<0.0001$ indicates significant differences between two groups as determined using Chi-square (and Fisher's exact) test. "bla $a_{\mathrm{KPC}}$-positive", proportion of KPC-positive isolates; "RM-", type I R-M systems negative; "RM+", type I R-M systems positive; "chromosome", organisms harboring type I R-M systems that are located in the bacterial chromosome; "plasmid-borne", organisms only harboring type I R-M systems that are carried by plasmids. Note: Organisms that only carried the type I R-M systems linked with plasmids were counted in the plasmid-borne group.

strains (Fig. 2 and Fig. S2, Supplementary data 1). In the type IA/B family, the clade 3 system was flanked by an integrase; the clade 2 system was linked with an $A B C$ transporter; clade 4, 6 and 7 systems, belonging to the type IC family, were carried by conjugative plasmids (Fig. 2). Moreover, clade 4 and 6 systems were also flanked by IS elements. Notably, these elements found linked with the type I R-M systems not only conferred the type I R-M unit with mobility, sometimes they increased the ability of restriction. The anticodon nuclease and ATP-dependent DNA helicase RecG identified in the clade 9 system contributed to DNA degradation (Fig. 3). Overall, linkage of type I R-M systems with various gene elements would enhance their evolution and sometimes be beneficial.
3.4. Type I R-M recognition sites are diverse enough to target the invading elements

Recognition sites comprising two specific target recognition domains (TRDs) are the vital clues for type I R-M scanning and cleavage [7]. Previous studies have also shown that the difference in the distance between the bases that are the substrates for methylation enhanced the scope for type I R-M diversification [7,9]. Hence, these distances in $K$. pneumoniae organisms were summarized and analysed. In the target sequences for members of the type IA/B families, the adenine residues were separated by 8 or $9 \mathrm{bp}$, in the IC family by 6,7 or 9 bp and in the ID family by 7 or 8 bp 


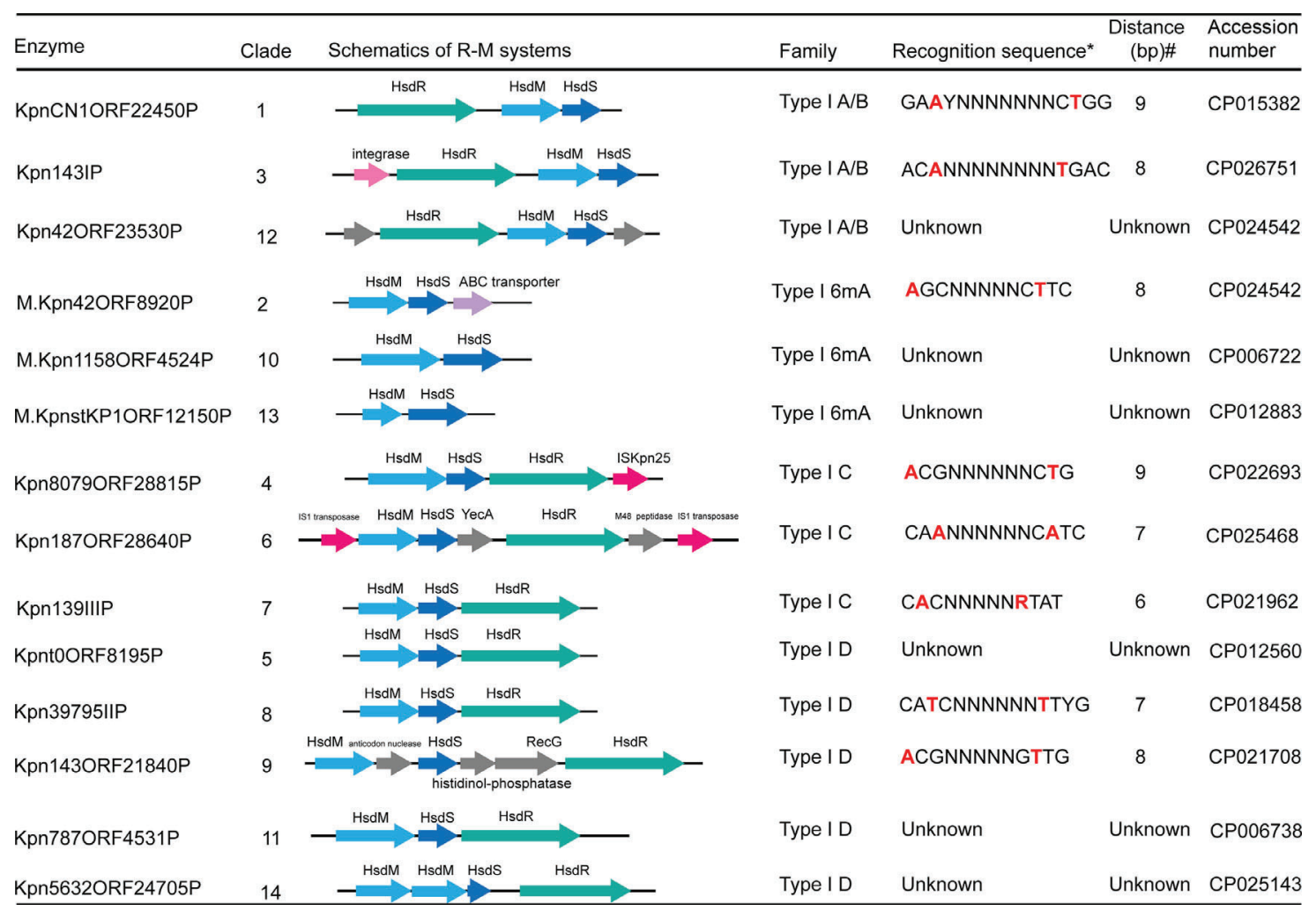

Fig. 2. The 14 major R-M units profiles identified in $\mathbf{K}$. pneumoniae. Genes in R-M systems are depicted as arrows in different colors. * N, any nucleotide; R, either purine; Y, either pyrimidine. Red bold type identifies either the adenine that is the target for methylation or the thymine complementary to the target adenine. \# No. base pairs between target adenines.

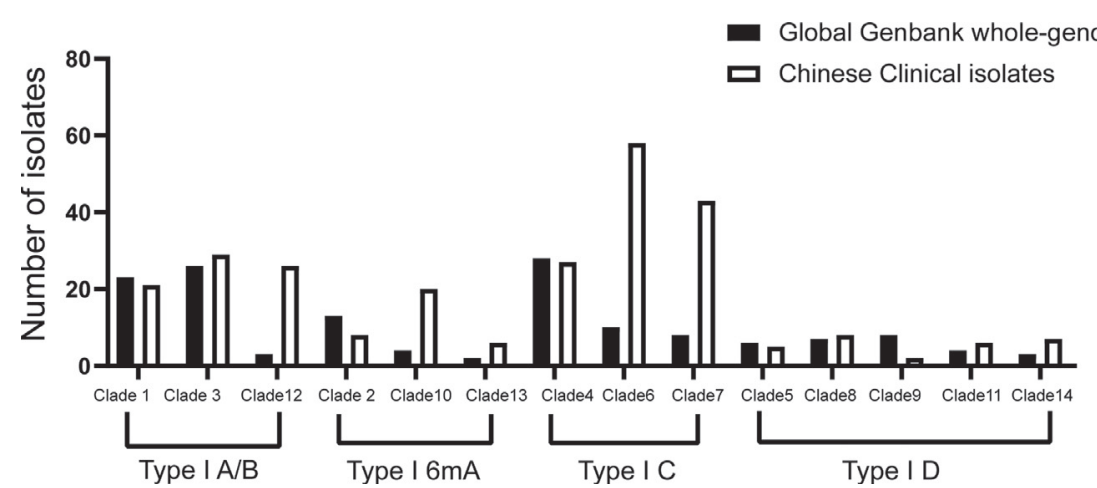

Fig. 3. The distribution of type I R-M systems in K. pneumoniae.

(Fig. 2). All the above findings indicated the recognition sites were diverse enough for type I R-M systems to function frequently in the genome of a K. pneumoniae organism at random. Moreover, the type I R-M systems can target more than 50 sites in a bla $a_{\mathrm{KPC}}$ plasmid (p187-2, CP025468.1, Table S3, Supplementary data 1), which revealed that such systems were associated with the dissemination

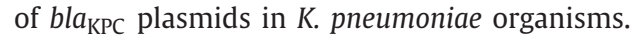

\subsection{Most type I R-M systems identified in $\mathrm{K}$. pneumoniae had effective anti-plasmid immunity}

The distribution of these systems among the isolates in Chinese clinical organisms and the global NCBI database were further investigated. The results indicated the predominant type I R-M family in clinical isolates and the global genome was type IC, which may be attributable to the linkage with mobile plasmids. The next most common was the type IA/B family, which was the fundamental sub-group of the type I R-M systems (Fig. 3). Notably, although there were some novel type I R-M units lacking REase, the prevalence of these units was low, indicating most type I R-M systems in K. pneumoniae could provide immunity against invading genetic elements that have not had sufficient exposure to the MTase of the cell, including the bla $a_{\mathrm{KPC}}$ genes.

\section{Discussion}

Several global epidemiological investigations have shown that the international high-risk KPC-producing K. pneumoniae (KPC-KP) contribute significantly to the sudden increase in MDR K. pneumoniae isolates $[15,16]$. However, little is known about the exact factors of successful KPC-KP dissemination. This study indicated the type I R-M systems may impact the spread of antibiotic resistance genes in K. pneumoniae populations.

Type I R-M systems are widespread and diverse [17], yet their correlation with the emergence of antibiotic-resistant $K$. pneumoniae lineages (KPC-KP) is not revealed. In the current study, 
type I R-M prevalence in $K$. pneumoniae was investigated and a highly significant inverse correlation was found between the presence of type I R-M systems and acquired antibiotic resistance (bla $a_{\mathrm{KPC}}$ gene) in $\mathrm{K}$. pneumoniae, particularly in high-risk CG258 lineages.

Although several plasmid-borne type I R-M, belonging to type IC units, were find in such MDR (KPC-KP and CG258 lineage) isolates, they showed high linkage to the resistance elements (e.g. $\left.b l a_{\mathrm{KPC}}\right)$. The linkage between R-M units and resistance plasmids indicated these systems would treat related resistant genes as selfDNA elements, not attacking (restriction), but protecting (methylation). These findings further verified the high-risk $K$. pneumoniae lineages were immunocompromised with loss of effective type I RM systems.

According to the epidemiological data of type I R-M systems in $K$. pneumonia, most systems possess the effective REase activity. Additionally, these systems identified in K. pneumoniae were pleomorphic with several elements adjunct, including mobile elements or other components that assist type I R-M systems. These evolutions of the RM structures enhanced the functional diversity of type I R-M systems, which would contribute to resist variable invading genetic elements. Moreover, all recognition sites identified in such systems were asymmetric and the spacing between the target adenines was heterogeneous. The findings showed the two TRDs recognized different sequences for the type I R-M systems in K. pneumonia, and the specificity can be changed by altering the spacing between target adenines. Hence, these systems appeared to be uniquely suited for diversification of sequence specificity.

Although the polymorphisms of type I R-M systems observed in K. pneumoniae indicated that such systems would have a significant impact on $b l a_{\mathrm{KPC}}$ acquisition, the type I R-M systems were not flawless barriers to plasmid transfer. In this study, several MDR groups (CG15, CG147 and CG29) could acquire several resistance genes, such as bla $a_{\mathrm{VIM}}, b l a_{\mathrm{IMP}}$ and bla $_{\mathrm{OXA}-48}$ [18], and successfully evaded restriction by R-M systems. These defense failures may be attributed to the pre-methylation of invading resistance genes or harboring anti-restriction proteins [6,19]. In addition to such innate immunity, another adaptive immunity, the CRISPR-Cas system (clustered, regularly interspaced palindromic repeat-CRISPR associated proteins) was also observed in bacterial strains [20]. Thus, once the invading MGEs escaped from the R-M immunity, organisms may acquire resistance to these infectious elements through other defense mechanisms, such as their CRISPR-Cas systems [20]. Further analysis of these "escapers" will be the focus of future work.

In summary, this work demonstrates the scarcity of type I R-M systems may be associated with the acquisition of bla $a_{\mathrm{KPC}}$ genes in K. pneumoniae. The lack of such systems makes it easier for KPC-KP to obtain $b l a_{\mathrm{KPC}}$ elements. Meanwhile, antibiotic use inadvertently selects for these strains with $b l a_{\mathrm{KPC}}$-plasmids, which may explain the KPC-KP epidemic success.

\section{Funding}

This work was supported by research grants from the National Natural Science Foundation of China [grant number 81871692 and 81572031] and by the University of Fribourg, Switzerland.

\section{Competing interests}

None to declare.

\section{Ethical approval}

Not required.

\section{Supplementary materials}

Supplementary material associated with this article can be found, in the online version, at doi:10.1016/j.ijantimicag.2020. 106050.

\section{References}

[1] McKenna M. Antibiotic resistance: the last resort. Nature 2013;499:394-6.

[2] Kuehn BM. Nightmare" bacteria on the rise in US hospitals, long-term care facilities. JAMA 2013;309:1573-4.

[3] Logan LK, Weinstein RA. The epidemiology of carbapenem-resistant Enterobacteriaceae: The impact and evolution of a global menace. J Infect Dis 2017;215:S28-36.

[4] Mathers AJ, Peirano G, Pitout JD. The role of epidemic resistance plasmids and international high-risk clones in the spread of multidrug-resistant Enterobacteriaceae. Clin Microbiol Rev 2015;28:565-91.

[5] Fu P, Tang Y, Li G, Yu L, Wang Y, Jiang X. Pandemic spread of blaKPC-2 among Klebsiella pneumoniae ST11 in China is associated with horizontal transfer mediated by IncFII-like plasmids. Int J Antimicrob Agents 2019;54:117-24.

[6] Goldberg GW, Marraffini LA. Resistance and tolerance to foreign elements by prokaryotic immune systems - curating the genome. Nat Rev Immunol $2015 ; 15: 717-24$

[7] Murray NE. Type I restriction systems: sophisticated molecular machines (a legacy of Bertani and Weigle). Microbiol Mol Biol Rev 2000;64:412-34.

[8] Titheradge AJ, King J, Ryu J, Murray NE. Families of restriction enzymes: an analysis prompted by molecular and genetic data for type ID restriction and modification systems. Nucleic Acids Res 2001;29:4195-205.

[9] Murray NE. 2001 Fred Griffith review lecture. Immigration control of DNA in bacteria: self-versus non-self. Microbiology 2002;148:3-20.

[10] Tock MR, Dryden DT. The biology of restriction and anti-restriction. Curr Opin Microbiol 2005;8:466-72.

[11] Luria SE, Human ML. A nonhereditary, host-induced variation of bacterial viruses. J Bacteriol 1952;64:557-69.

[12] Roberts RJ, Vincze T, Posfai J, Macelis D. REBASE-a database for DNA restriction and modification: enzymes, genes and genomes. Nucleic Acids Res 2015;43:D298-9

[13] Kumar S, Stecher G, Li M, Knyaz C, Tamura K. MEGA X: Molecular evolutionary genetics analysis across computing platforms. Mol Biol Evol 2018;35:1547-9.

[14] Chen L, Mathema B, Chavda KD, DeLeo FR, Bonomo RA, Kreiswirth BN. Carbapenemase-producing Klebsiella pneumoniae: molecular and genetic decoding. Trends Microbiol 2014;22:686-96.

[15] Tzouvelekis LS, Markogiannakis A, Psichogiou M, Tassios PT, Daikos GL. Carbapenemases in Klebsiella pneumoniae and other Enterobacteriaceae: an evolving crisis of global dimensions. Clin Microbiol Rev 2012;25:682-707.

[16] Peirano G, Bradford PA, Kazmierczak KM, Chen L, Kreiswirth BN, Pitout JD. Importance of clonal complex 258 and IncFK2-like plasmids among a global collection of Klebsiella pneumoniae with blaKPC. Antimicrob Agents Chemother 2017:61(4):e02610-16.

[17] Oliveira PH, Touchon M, Rocha EP. The interplay of restriction-modification systems with mobile genetic elements and their prokaryotic hosts. Nucleic Acids Res 2014;42:10618-31.

[18] David S, Reuter S, Harris SR, Glasner C, Feltwell T, Argimon S, et al. Epidemic of carbapenem-resistant Klebsiella pneumoniae in Europe is driven by nosocomial spread. Nat Microbiol 2019;4:1919-29.

[19] Vasu K, Nagaraja V. Diverse functions of restriction-modification systems in addition to cellular defense. Microbiol Mol Biol Rev 2013;77:53-72.

[20] Zhou Y, Tang Y, Fu P, Tian D, Yu L, Huang Y, et al. The type I-E CRISPR-Cas system influences the acquisition of blaKPC-IncF plasmid in Klebsiella pneumonia. Emerg Microbes Infec 2020;9:1011-22. 